
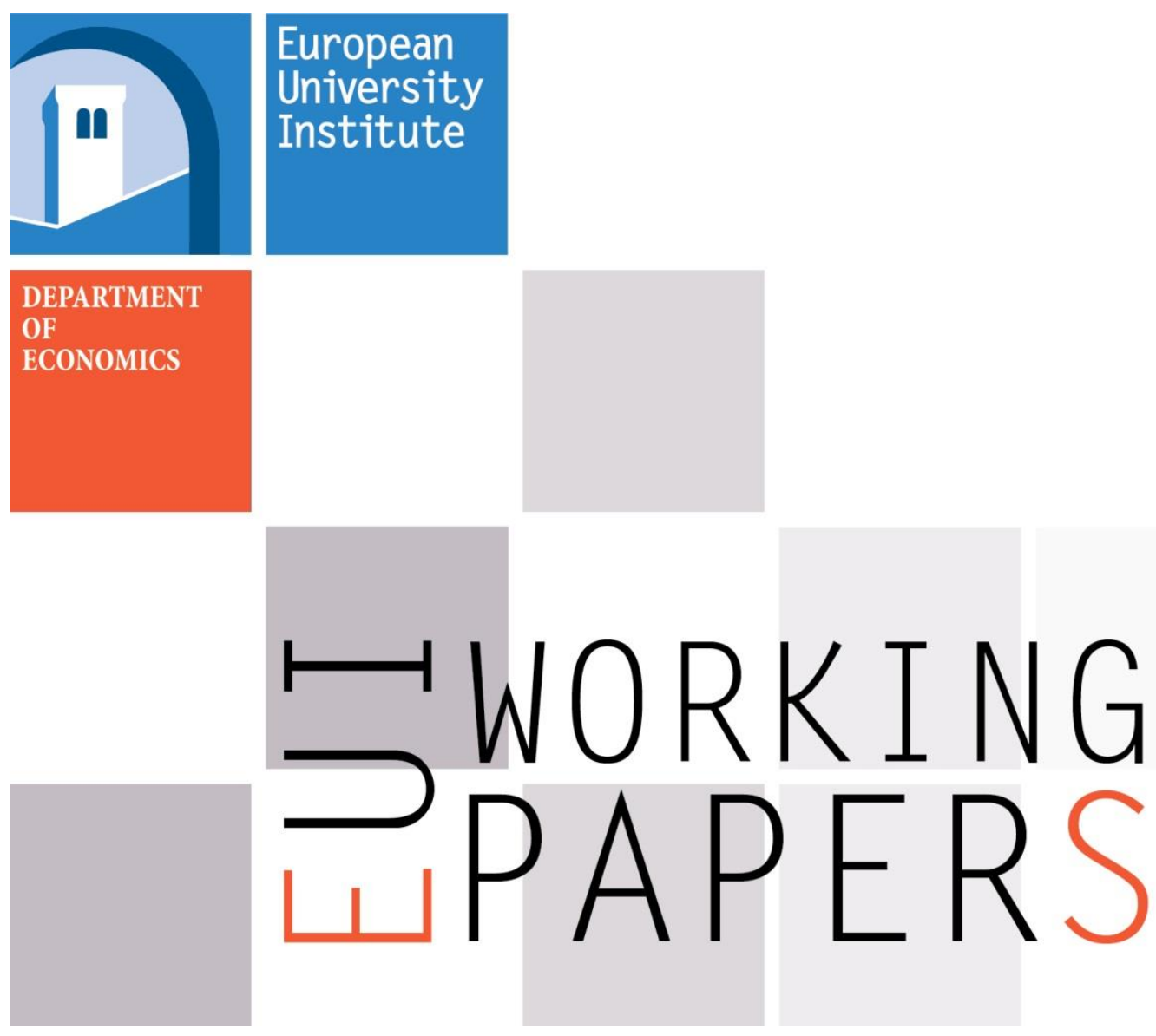

ECO 2015/02

Department of Economics

EXISTENCE AND UNIQUENESS OF EQUILIBRIUM IN LUCAS' ASSET PRICING MODEL WHEN UTILITY IS UNBOUNDED

João Brogueira and Fabian Schütze 

European University Institute

Department of Economics

Existence and uniqueness of equilibrium in Lucas' Asset Pricing model when utility is unbounded

João Brogueira and Fabian Schütze

EUI Working Paper ECO 2015/02 
This text may be downloaded for personal research purposes only. Any additional reproduction for other purposes, whether in hard copy or electronically, requires the consent of the author(s), editor(s). If cited or quoted, reference should be made to the full name of the author(s), editor(s), the title, the working paper or other series, the year, and the publisher.

ISSN 1725-6704

(C) João Brogueira and Fabian Schütze, 2015

Printed in Italy

European University Institute

Badia Fiesolana

I - 50014 San Domenico di Fiesole (FI)

Italy

www.eui.eu

cadmus.eui.eu 


\title{
Existence and uniqueness of equilibrium in Lucas' Asset Pricing model when utility is unbounded*
}

\author{
João Brogueira and Fabian Schütze ${ }^{\dagger}$
}

April 2, 2015

\begin{abstract}
This note proves existence of a unique equilibrium in a Lucas (1978) economy when the utility function displays constant relative risk aversion and log dividends follow a normally distributed AR(1) process with positive auto-correlation. In particular, the note provides restrictions on the coefficient of relative risk aversion, the discount factor and the conditional variance of the consumption process that ensure existence of a unique equilibrium.
\end{abstract}

Keywords: Asset Pricing, Exchange Economy, Dynamic Programming, Equilibrium Conditions.

JEL: C61, C62, D51, G12.

\section{Introduction}

This note proves existence of a unique equilibrium in a Lucas (1978) economy when the utility function displays constant relative risk aversion (CRRA)

*This note evolved out of a conversation with Thomas Sargent and John Stachurski. We want to thank them for constant encouragement and especially John Stachurski for detailed comments on earlier drafts. We are grateful to Ramon Marimon for invaluable advice during the entire process of writing this note.

$\dagger$ Both authors are at the European University Institute, Florence. Corresponding author: joao.brogueira@eui.eu. 
and log dividends follow a normally distributed $\mathrm{AR}(1)$ process with positive auto-correlation. The equilibrium in the economy is characterized by a pricing function for the Lucas-tree and a value function for the representative consumer. Under the assumption of a bounded utility function, Lucas proves existence of a unique equilibrium by showing that the pricing and value functions are fixed points of functional equations. Lucas resorted to the sufficient conditions of Blackwell (1965) to document that Banach's fixed point theorem guarantees existence of a unique solution to the functional equations. Alas, Blackwell's conditions do not hold when the utility function displays the CRRA property. The conditions require utility being bounded in the sup-norm, which does not hold when the consumption space is equal to the positive real numbers and the investor displays CRRA preferences. Fortunately, Blackwell's conditions are only sufficient. We exploit the extensions of Blackwell's conditions by Boyd (1990) and document under which circumstances Banach's theorem can be applied. In particular, we provide a joint restriction on the coefficient of relative risk aversion, the discount factor and the conditional variance of the consumption process that ensure existence of a unique equilibrium. This paper is a complement to Kamihigashi (1998). He proved that any equilibrium in a Lucas-type economy with CRRA utility is unique. Our paper documents under which conditions such an equilibrium exists. Alvarez and Stokey (1998) analyses dynamic programming problems with homogeneous return functions and transition functions that are homogeneous of degree one. In this note we use similar arguments as they do and consider a transition function that is not homogeneous of degree one. The outline of this note is as follows. In Section 2 we provide a brief description of the economy and the definition of equilibrium in the asset pricing problem. Section 3 presents a restriction on the parameter space that ensures existence and uniqueness of equilibrium and discusses an extension to Blackwell's sufficient conditions for a given operator on a metric space to be a contraction that is useful to our argument. We establish existence and uniqueness of equilibrium and study properties of the pricing function in Section 4. Section 5 contains concluding comments.

\section{The economy and definition of equilibrium}

This note describes the equilibrium in the same way as Lucas does. Denote by $x^{\prime} \in X$ next period's share holdings, with $X=[0, \bar{x}], \bar{x}>1$ and current 
consumption by $c$. Let $y \in Y=\mathbf{R}_{++}$be the current dividend of a Lucastree. The transition equation for next period's dividend is $G\left(y, z^{\prime}\right)=y^{\alpha} z^{\prime}$ with $\alpha \in(0,1)$ and $\log z^{\prime} \sim N\left(0, \sigma^{2}\right)$. Let $Q$ be the probability density over $z^{\prime}$. Instantaneous utility is given by $u(c)=c^{1-\gamma} /(1-\gamma), \gamma>0$. An equilibrium is formally defined as:

Definition 1. An equilibrium is a continuous function $p(y): Y \rightarrow \mathbf{R}_{+}$and a continuous function $v(y, x): Y \times X \rightarrow \mathbf{R}_{+}$such that:

$$
v(y, x)=\max _{c, x^{\prime} \in \Gamma(y, x)}\left\{u(c)+\beta \int_{Z} v\left(G\left(y, z^{\prime}\right), x^{\prime}\right) Q\left(d z^{\prime}\right)\right\}
$$

with

$$
\Gamma(y, x)=\left\{\left(c, x^{\prime}\right) \in Y \times X: c+p(y) x^{\prime} \leq y x+p(y) x\right\}
$$

and

$$
\text { for each } y, v(y, 1) \text { is attained by } c=y \text { and } x^{\prime}=1 \text {. }
$$

\section{Sufficient conditions for the existence of a unique equilibrium}

The following proposition states under which conditions a unique equilibrium exists:

Proposition 1. Take $\beta \in(0,1), \sigma \in(0, \infty)$.

(A1) Suppose that for $\gamma \in(0,1)$ :

$$
\beta\left[0.5+\int_{1}^{\infty}\left(z^{\prime}\right)^{1-\gamma} Q\left(d z^{\prime}\right)\right]<1 .
$$

(A2) Suppose that for $\gamma>1$ :

$$
\beta\left[\int_{0}^{1}\left(z^{\prime}\right)^{1-\gamma} Q\left(d z^{\prime}\right)+0.5\right]<1 .
$$

Then there exists a unique equilibrium. That is:

(i) There exists a unique non-negative continuous pricing function $p(y)$. 
(ii) There exists a unique non negative function $v(y, x)$.

Inequalities $(A 1)$ and $(A 2)$ will be described at the end of this section. Given a value function $v$, we first study existence of a unique pricing function. Assume that for each $y, v(y, x)$ is an increasing, concave and differentiable function with respect to $x$. The first order conditions of the representative agent's maximization problem are:

$$
\begin{aligned}
\frac{\partial u(c)}{\partial c} p(y) & =\beta \int_{Z} \frac{\partial v\left(G\left(y, z^{\prime}\right), x^{\prime}\right)}{\partial x^{\prime}} Q\left(d z^{\prime}\right) \\
c+p(y) x^{\prime} & =y x+p(y) x
\end{aligned}
$$

The derivative of $v$ with respect to $x^{\prime}$ is:

$$
\frac{\partial v\left(G\left(y, z^{\prime}\right), x^{\prime}\right)}{\partial x^{\prime}}=\frac{\partial u\left(G\left(y, z^{\prime}\right)\right)}{\partial y^{\prime}}\left[G\left(y, z^{\prime}\right)+p\left(G\left(y, z^{\prime}\right)\right)\right]
$$

Using the condition above, defining $f(y)=p(y) \partial u(y) / \partial y$ and using the equilibrium conditions $x=x^{\prime}=1$ and $c=y$ allows reformulating the stochastic Euler equation (2) as:

$$
f(y)=h(y)+\beta \int_{Z} f\left(G\left(y, z^{\prime}\right)\right) Q\left(d z^{\prime}\right),
$$

with $h(y)=\beta \int_{Z}\left[\frac{\partial u\left(G\left(y, z^{\prime}\right)\right)}{\partial y^{\prime}} G\left(y, z^{\prime}\right)\right] Q\left(d z^{\prime}\right)=\beta y^{\alpha(1-\gamma)} \exp \left((1-\gamma)^{2} \sigma^{2} / 2\right)$.

Lucas uses Blackwell's sufficient conditions to show that the operator $T$, defined such that (3) is equivalent to $T f=f$, is a contraction and then applies Banach's fixed point theorem. To employ Blackwell's conditions, Lucas assumes that the utility function $u$ and thereby the function $h$ is bounded with the sup-norm. Unfortunately, with CRRA utility and a dividend process in $\mathbf{R}_{++}$, the function $h$ is unbounded with the sup-norm. Importantly, boundedness is a characteristic that is closely linked to the employed metric. In the following subsection we study a norm with respect to which the function $h$ in (3) is bounded.

\subsection{How to guarantee uniqueness of the equilibrium}

Boyd (1990) extended Blackwell's sufficient conditions by generalizing the metric from a sup-norm to a weighted sup-norm. Denote the set of continuous 
functions $f: Y \rightarrow \mathbf{R}_{+}$by $S$. Take $\varphi \in S, \varphi>0$. Then $f$ is $\varphi$-bounded with respect to the weighted sup-norm $\|f\|_{\varphi}=\sup _{y \in Y}\{|f(y)| / \varphi(y)\}$ if $\exists B \in \mathbf{R}_{+}$ such that $\|f\|_{\varphi}<B$. Let $S_{\varphi} \subset S$ be the set of continuous and $\varphi$-bounded functions. Note that $\left(S_{\varphi}, \varphi\right)$ is a complete metric space. Boyd's sufficient conditions are:

Lemma 1 (Boyd's sufficient conditions). Let $T: S_{\varphi} \rightarrow S$ and suppose:

(B1) (monotonicity) $T$ is monotone, that is $\forall f, g \in S_{\varphi}, f \geq g$ implies $T f \geq$ $\mathrm{Tg}$

(B2) (discounting) For any $A \in \mathbf{R}_{++}$, there exists $\theta \in(0,1)$ such that: $T(f+A \varphi) \leq T f+\theta A \varphi$;

(B3) (self-map) $T(0) \in S_{\varphi}$.

Then $T$ is a contraction with modulus $\theta$.

The discounting and self-map property involve the weighting function explicitly. The self-map property requires the function $h$ to be bounded with a weighted sup-norm. The restriction implied by the discounting property is satisfied if the following inequality holds:

$$
\beta \frac{\int_{Z} \varphi\left(G\left(y, z^{\prime}\right)\right) Q\left(d z^{\prime}\right)}{\varphi(y)}<1 .
$$

Similarly to the discussion of dynamic programming techniques with homogeneous return functions in Alvarez and Stokey (1998), the inequality places a bound on the expected growth rate of the weighting function. Some intuition for the weight $\varphi$ we will use can be gleaned from studying the ratio in (4). Let $\gamma \in(0,1)$; then for all $z^{\prime} \in\left(0, y^{-\alpha}\right), y^{1-\gamma}<G\left(y, z^{\prime}\right)^{1-\gamma}$. Furthermore for $z^{\prime}>y^{-\alpha}, G\left(y, z^{\prime}\right)^{1-\gamma}<y^{1-\gamma}$. The integral of the function $G\left(y, z^{\prime}\right)^{1-\gamma}$ over $Q$ (times $\beta$ ) is equivalent to the function $h$. This observation suggests that $h$ can be bounded with:

$$
\varphi(y)=\max \left\{1, y^{1-\gamma}\right\}
$$


More explicitly, using (5) the left hand side of (4) satisfies (yet $\gamma \in(0,1)$ ):

$$
\begin{aligned}
& \beta \frac{\int_{Z} \varphi\left(G\left(y, z^{\prime}\right)\right) Q\left(d z^{\prime}\right)}{\varphi(y)}=\beta \frac{\int_{0}^{y^{-\alpha}} Q\left(d z^{\prime}\right)+y^{\alpha(1-\gamma)} \int_{y^{-\alpha}}^{\infty}\left(z^{\prime}\right)^{1-\gamma} Q\left(d z^{\prime}\right)}{\varphi(y)} \\
& \leq\left\{\begin{array}{cl}
\frac{\beta\left[\int_{0}^{y^{-\alpha}} Q\left(d z^{\prime}\right)+\int_{y^{-\alpha}}^{\infty} z^{\prime 1-\gamma} Q\left(d z^{\prime}\right)\right]}{1} & \text { if } 0<y<1 \\
\frac{y^{\alpha(1-\gamma)} \beta\left[\int_{0}^{y^{-\alpha}} Q\left(d z^{\prime}\right)+\int_{y^{-\alpha}}^{\infty} z^{1-\gamma} Q\left(d z^{\prime}\right)\right]}{y^{(1-\gamma)}} & \text { if } y \geq 1
\end{array}\right. \\
& \leq \beta\left[\int_{0}^{y^{-\alpha}} Q\left(d z^{\prime}\right)+\int_{y^{-\alpha}}^{\infty} z^{1-\gamma} Q\left(d z^{\prime}\right)\right] \quad \forall y \in Y \\
& \leq \beta\left[\int_{0}^{1} Q\left(d z^{\prime}\right)+\int_{1}^{\infty} z^{1-\gamma} Q\left(d z^{\prime}\right)\right] \quad \forall y \in Y \\
& =\beta\left[0.5+\int_{1}^{\infty} z^{1-\gamma} Q\left(d z^{\prime}\right)\right] \quad \forall y \in Y
\end{aligned}
$$

In the numerator of the first equality, the integral is split into two subdomains and the weighting function is written explicitly. The inequalities in the second line hold as $1>y^{\alpha(1-\gamma)}$ for $0<y<1$ and $y^{\alpha(1-\gamma)} \geq 1$ for $y \geq 1$. The inequality in the second line follows as $y^{\alpha(1-\gamma)}<y^{1-\gamma}$ for $y>1$. Finally, the last inequality holds because the term in square brackets is maximized at $y=1$. If the condition in (A1) is fulfilled, the argument above shows that the weighting function (5) guarantees that (B2) holds. (A1) and (A2) represent a restriction on the parameters $(\sigma, \gamma, \beta)$. The shaded region in Figure 1 documents which parameter pairs $(\sigma, \gamma)$ satisfy the conditions in Proposition 1 when $\beta=0.99$. When $\gamma=1, u(c)=\log (c)$ and a solution to (6) can be calculated analytically. Figure 1 reveals that the admissible region for $\sigma$ increases when $\gamma \rightarrow 1$. Lower values of $\beta$ enlarge the admissible region. In the following, the proof of Part $i$ of Proposition 1 will be completed.

\section{Proof of existence and uniqueness of equi- librium.}

In this section we formally prove existence and uniqueness of a pricing function in the economy described in Section 2. 

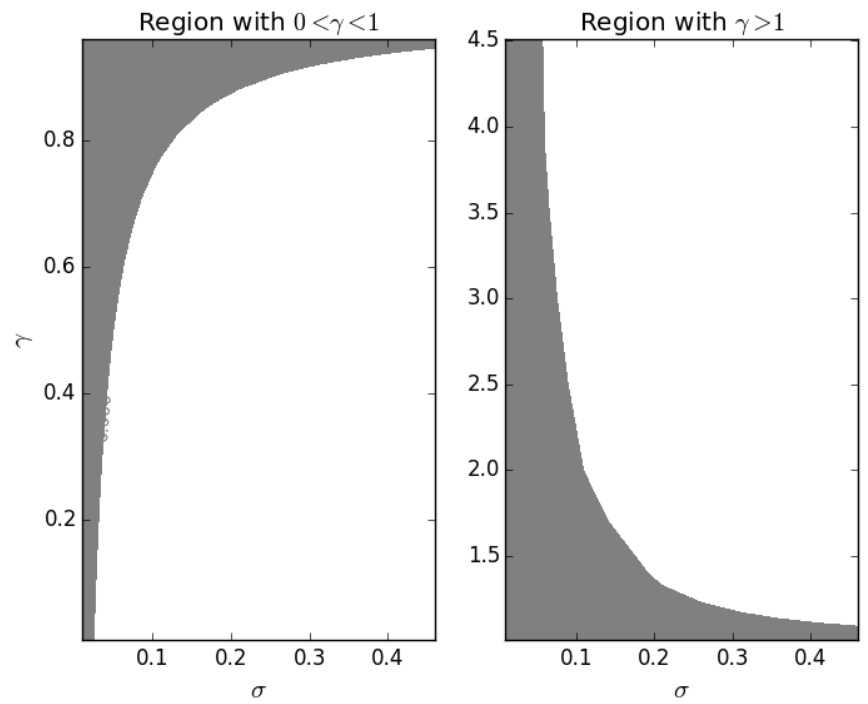

The shaded region represent the area combinations of $(\gamma, \sigma)$ satisfying the restriction of Proposition 1. The white region documents combinations of $(\gamma, \sigma)$ violating the restriction.

Figure 1: Contour plots for the set of $(\gamma, \sigma)$ satisfying the Conditions in Proposition 1.

Proof. (Part (i) of Proposition 1.) The proof begins by showing that $T$ : $S_{\varphi} \rightarrow S$. As both $h$ and the integral over $f$ are continuous, $T f$ is continuous. Hence $T: S_{\varphi} \rightarrow S$. The operator $T$ is monotone since for any $f \geq g, \int f\left(G\left(y, z^{\prime}\right)\right) Q\left(d z^{\prime}\right) \geq \int g\left(G\left(y, z^{\prime}\right)\right) Q\left(d z^{\prime}\right)$, so $T f \geq T g$ and (B1) holds. Under assumption (A1) condition (B2) is satisfied for $0<\gamma<1$, as the argument in section 2.2 shows. It remains to be shown that this condition 
holds for $\gamma>1$. For these values of $\gamma$, we observe the following:

$$
\begin{aligned}
& \beta \frac{\int_{Z} \varphi\left(G\left(y, z^{\prime}\right)\right) Q\left(d z^{\prime}\right)}{\varphi(y)}=\beta\left[y^{\alpha(1-\gamma)} \int_{0}^{y^{-\alpha}} z^{1-\gamma} Q\left(d z^{\prime}\right)+\int_{y^{-\alpha}}^{\infty} Q\left(d z^{\prime}\right)\right] \\
& \leq\left\{\frac{y^{\alpha(1-\gamma)}\left[\int_{0}^{y^{-\alpha}} z^{1-\gamma} Q\left(d z^{\prime}\right)+\int_{y^{-\alpha}}^{\infty} Q\left(d z^{\prime}\right)\right]}{y^{1-\gamma}} \text { if } 0<y<1,\right. \\
& \leq \beta\left[\int_{0}^{y^{-\alpha}} z^{1-\gamma} Q\left(d z^{\prime}\right)+\int_{y^{-\alpha}}^{\infty} Q\left(d z^{\prime}\right)\right] \\
& \text { if } y \geq 1 . \\
& \leq \beta\left[\int_{0}^{11-\gamma} Q\left(d z^{\prime}\right)+\int_{y^{-\alpha}}^{\infty} Q\left(d z^{\prime}\right)\right] \forall y \in Y \\
&=\beta\left[\int_{0}^{1} z^{\prime 1-\gamma} Q\left(d z^{\prime}\right)+0.5\right]
\end{aligned}
$$

The reasoning for each condition is analogue to the one made for the case $0<\gamma<1$. Under (A2), condition (B2) holds for $\gamma>1$. The third condition of Lemma 1 requires $h$ to be bounded with the weighted sup-norm. Hence, since:

$$
\|h\|_{\varphi}=\beta \exp \left((1-\gamma)^{2} \sigma^{2} / 2\right) \sup _{y \in Y}\left\{\frac{y^{\alpha(1-\gamma)}}{\varphi(y)}\right\}=\beta \exp \left((1-\gamma)^{2} \sigma^{2} / 2\right),
$$

$T(0) \in S_{\varphi}$ and (B3) of Lemma 1 holds. Concluding, $T$ is a contraction and by Banach's fixed point theorem a unique function $f$ satisfying (3) exists. The solution is non-negative and continuous. Therefore the pricing function $p(y)=f(y) / u^{\prime}(y)$ is continuous and non-negative as well.

After having shown that there exists a unique pricing function $p$ given $v$, the converse remains to be shown. The following argument completes the proof of Proposition 1.

Proof. (Part (ii) of Proposition 1). Define the operator $H$ such that:

$$
H v(y, x)=\max _{c, x^{\prime} \in \Gamma(y, x)}\left\{u(c)+\beta \int_{Z} v\left(G\left(y, z^{\prime}\right), x^{\prime}\right) Q\left(d z^{\prime}\right)\right\},
$$


To prove that a unique fixed point $H v=v$ exists, one can resort to Lemma 1 to show that $H$ is a contraction and then use Banach's theorem to establish existence and uniqueness of the fix point. Begin by showing $H: S_{\varphi} \rightarrow S$. As before, $u(c)+\beta \int_{Z} v\left(G\left(y, z^{\prime}\right), x\right) Q\left(d z^{\prime}\right)$ is continuous. Since for each $y$, the budget correspondence is compact valued and continuous, Berge's theorem guarantees that $H v$ is continuous. Hence $H: S_{\varphi} \rightarrow S$. Monotonicity of $H$ holds. The weighting function can be defined similarly as:

$$
\varphi(y, x)=\varphi(y)=\max \left\{1, y^{1-\gamma}\right\} .
$$

Discounting of $H$ can thus be established as in the proof of Part $(i)$ of Proposition 1 , above. Finally, one needs to show that $H$ is a self-map. In mathematical terms, $H(0) \in S_{\varphi}$ if:

$$
\sup _{(y, x) \in Y \times X}\left\{\frac{\left|\max _{c, x^{\prime} \in \Gamma(y, x)} u(c)\right|}{\varphi(y, x)}\right\}<+\infty
$$

By the definition of the weighting function $\left\|\max _{c, x^{\prime} \in \Gamma(y, x)} u(c)\right\|_{\varphi}=|1-\gamma|$. Hence $H$ is a contraction and Banach's fixed point theorem establishes that it has a unique solution.

At this stage, a characterization of function $f$ that solves (3) is in order. The following Lemma documents some of its properties:

\section{Lemma 2.}

1. For any $f_{0} \in S_{\varphi}, \lim _{n \rightarrow \infty} T^{n} f_{o}=f$.

2. Suppose $0<\gamma<1$. Then, both $h$ and $f$ are strictly increasing and concave. Suppose otherwise $1<\gamma$. Then both $h$ and $f$ are strictly decreasing and convex.

Proof. Point 1 of Lemma 2 follows directly from the fact that $T$ is a contraction and hence will not be proved here. Point 2 is proved in the Appendix to this note.

Properties of $v$ assumed in section 2.1 can be shown by arguments similar to Lucas (1978) (in propositions 1 and 2). This concludes the proof of Proposition 1. 


\section{Conclusion}

This paper presents a proof of existence and uniqueness of equilibrium in a pure exchange economy of Lucas (1978), when the utility function takes the CRRA form and the dividend stream follows an autoregressive process of order one with positive autocorrelation. We leave for further research the extension of our proof to the case in which innovations affect the growth rate of dividends, instead of the level, a common specification of Lucas' asset pricing model.

\section{Appendix}

Proof. (Part ii of Lemma 1) As in the text, denote by $S_{\varphi}$ the set of continuous and $\varphi$-bounded functions. The set $S_{\varphi}^{\prime}$ is the set of continuous, $\varphi$-bounded, nondecreasing and concave functions, and $S_{\varphi}^{\prime \prime} \subset S_{\varphi}^{\prime}$ imposes additionally strict monotonicity and concavity. We want to show that the contraction operator $T$ maps any function $\tilde{f} \in S_{\varphi}^{\prime}$ into the subset $S_{\varphi}^{\prime \prime}$. As the solution to the functional equation is characterized by $T f=f$ and $S_{\varphi}^{\prime}$ is a closed set, if the operator $T$ transforms any nondecreasing and concave function into a strictly increasing and concave function, then $f$ is strictly increasing and concave (Corollary 1 of the Contraction Mapping Theorem in Stokey and Lucas (1989), p.52). To show the desired result, suppose first that $h$ is strictly increasing and concave and pick any $\tilde{f} \in S_{\varphi}^{\prime}$. To begin, study whether $T \tilde{f}$ is strictly increasing. For any pair $\hat{y}, y \in Y$ with $\hat{y}>y$, the function $T \tilde{f}$ satisfies:

$$
\begin{aligned}
T \tilde{f}(\hat{y}) & =h(\hat{y})+\beta \int_{Z} \tilde{f}\left(G\left(\hat{y}, z^{\prime}\right)\right) Q\left(d z^{\prime}\right) \\
& >h(y)+\beta \int_{Z} \tilde{f}\left(G\left(y, z^{\prime}\right)\right) Q\left(d z^{\prime}\right) \\
& =T \tilde{f}(y) .
\end{aligned}
$$

The inequality holds because $G$ and $h$ are strictly increasing and $\tilde{f}$ is nondecreasing. Hence, $T \tilde{f}$ is strictly increasing. To analyze concavity, define $y_{\omega}=\omega y+(1-\omega) y^{\prime}$, for any $y, y^{\prime} \in Y, y \neq y^{\prime}$, and $0<\omega<1$. The strict 
concavity form of $h$ and $G$, together with $\tilde{f}$ being concave, ensure that:

$$
\begin{aligned}
T \tilde{f}\left(y_{\omega}\right) & =h\left(y_{\omega}\right)+\beta \int_{Z} \tilde{f}\left(G\left(y_{\omega}, z^{\prime}\right)\right) Q\left(d z^{\prime}\right) \\
& >\omega\left[h(y)+\beta \int_{Z} \tilde{f}\left(G\left(y, z^{\prime}\right)\right) Q\left(d z^{\prime}\right)\right]+(1-\omega)\left[h\left(y^{\prime}\right)+\beta \int_{Z} \tilde{f}\left(G\left(y^{\prime}, z^{\prime}\right)\right) Q\left(d z^{\prime}\right)\right] \\
& =\omega T \tilde{f}(y)+(1-\omega) T \tilde{f}\left(y^{\prime}\right) .
\end{aligned}
$$

The function $T \tilde{f}$ is strictly concave. Taken together, we know that for any $\tilde{f} \in S_{\varphi}^{\prime}, T \tilde{f} \in S_{\varphi}^{\prime \prime}$. Hence, $f$ must be an element of the set $S_{\varphi}^{\prime \prime}$, guaranteeing that $f$ has the same functional form as $h$. Now, suppose $h$ is convex and falling. We could again define the operator $T$ as $T f(y)=$ $h(y)+\beta \int_{Z} f\left(G\left(y, z^{\prime}\right)\right) Q\left(d z^{\prime}\right)$ and study into which subset a candidate solution is mapped into. To facilitate analysis though, take a different route. Look at the modified operator $T f_{-}=h_{-}+\beta \int_{Z} f_{-}\left(G\left(y, z^{\prime}\right)\right) Q\left(z^{\prime}\right)$, with $h_{-}=-h$ and $f_{-}=-f$. Under the same assumptions guaranteeing a unique solution to the original contraction mapping, there exists a unique solution to the modified contraction mapping. As $h_{-}$is strictly increasing and concave, the proof above applies to the modified contraction mapping. As $f_{-}$is strictly increasing and concave, $f$ is strictly decreasing and convex and inherits the properties of $h$.

\section{References}

Fernando Alvarez and Nancy L Stokey. Dynamic programming with homogeneous functions. Journal of Economic Theory, 82(1):167-189, 1998.

David Blackwell. Discounted dynamic programming. The Annals of Mathematical Statistics, 36(1):226-235, 1965.

John III Boyd. Recursive utility and the ramsey problem. Journal of Economic Theory, 50(2):326-345, 1990.

Takashi Kamihigashi. Uniqueness of asset prices in an exchange economy with unbounded utility. Economic Theory, 12(1):103-122, 1998.

Robert E. Lucas, Jr. Asset prices in an exchange economy. Econometrica, 46(6):1429-1445, 1978.

Nancy L. Stokey and Robert E. Lucas. Recursive methods in economic dynamics. Harvard University Press, Cambridge, Mass, 1989. 